\title{
Molecular dynamics and forces of a motile cell simultaneously visualized by TIRF and force microscopies
}

\author{
Yoshiaki Iwadate and Shigehiko Yumura \\ Department of Functional Molecular Biology, Graduate School of Medicine, \\ Yamaguchi University, Yamaguchi, Japan \\ BioTechniques 44:739-750 (May 2008) \\ doi $10.2144 / 000112752$
}

Cells must exert traction forces onto the substratum for continuous migration. Molecular dynamics such as actin polymerization at the front of the cell and myosin II accumulation at the rear should play important roles in the exertion of forces required for migration. Therefore, it is important to reveal the relationships between the traction forces and molecular dynamics. Traction forces can be calculated from the deformation of the elastic substratum under a migrating cell. A transparent and colorless elastic substratum with a high refractive index (1.40) and a low Young's modulus $(1.0 \mathrm{kPa})$ were made from a pair of platinum-catalyzed silicones. We used this substratum to develop a new method for simultaneous recording of molecular dynamics and traction forces under a migrating cell in which total internal refractive fluorescence (TIRF) and force microscopies were combined. This new method allows the detection of the spatiotemporal distribution of traction forces produced by individual filopodia in migrating Dictyostelium cells, as well as simultaneous visualization of these traction forces and the dynamics of filamentous myosin II.

\section{INTRODUCTION}

Cell migration plays an essential role in the development of most organisms $(1,2)$ and in adult life (3). A migrating cell exerts the traction forces to the substratum to realize its migration. Traction forces are generated by intracellular molecular machinery. At the anterior of the cell, actin polymerization is considered the source of the driving forces for extension of the leading edge (3-6). On the other hand, the detachment of the rear of the cell from the substratum and its retraction is thought to occur through contraction via a myosin II-dependent process (7-11). At the front of the cell, actin polymerizationmediated counterforces should therefore be exerted onto the substratum in the opposite direction of extension. On the other hand, prior to detachment of the rear of the cell from the substratum, myosin II accumulation and a subsequent increase in traction forces should be exerted onto the substratum via a myosin II-dependent process. To detect the traction forces exerted by migrating cells, Dembo and colleagues $(12,13)$ developed force microscopy, in which the measurement of deformation of the elastic substratum under a migrating cell was converted into stress in the substratum using finite element methods. They showed a detailed map under migrating fibroblasts (14-19) and revealed dynamic traction stresses at the leading edge of migrating fibroblasts $(15,16)$. On the other hand, Butler et al. (20) proposed another kind of calculation method, namely Fourier-transform traction cytometry, and showed a detailed traction map under smooth muscle cells.

Fluorescence of green fluorescent protein (GFP)-zyxin in a migrating fibroblast and traction forces were simultaneously observed using fluorescence microscopy (17), and in some cases, traction forces appeared at nascent focal adhesions. Simultaneous observation of more detailed molecular dynamics, such as polymerization of actin at the leading edge or accumulation of filamentous myosin II at the rear end of migrating cells, as well as exertion of traction forces onto the substratum, could provide important information about the relationships between molecular dynamics and their production of traction forces.
Dictyostelium is one of the most appropriate materials to investigate the relationships between cell migration and molecular dynamics because Dictyostelium cells are known as fast moving irregularly shaped cells and molecular genetic approaches can be easily applied to them, such as expressing GFP fusion proteins. Although it had been difficult to measure the traction force of migrating Dictyostelium cells because of their small size, several authors recently showed the distribution of traction forces under a migrating Dictyostelium cell (11,20-23).

We therefore developed a new method for simultaneous recording of molecular dynamics and traction forces under migrating cells, in which total internal refractive fluorescence (TIRF) and force microscopy were combined. Here we describe the method and its application to simultaneous recording of enhanced GFP-myosin II and traction forces under a migrating Dictyostelium cell, as well as the recording of traction forces produced by individual filopodia.

\section{MATERIALS AND METHODS}

\section{Cell Culture}

Dictyostelium discoideum cells were cultured in HL5 medium $(1.3 \% \mathrm{w} / \mathrm{v}$ bacteriological peptone, $0.75 \% \mathrm{w} / \mathrm{v}$ yeast extract, $85.5 \mathrm{mM}$ D-glucose, $3.5 \mathrm{mM}$ $\mathrm{Na}_{2} \mathrm{HPO}_{4} 12 \mathrm{H}_{2} \mathrm{O}, 3.5 \mathrm{mM} \mathrm{KH} \mathrm{PO}_{4}, \mathrm{pH}$ 6.4) and developed until the cells became aggregation-competent in a balanced salt solution (BSS; $10 \mathrm{mM} \mathrm{NaCl}, 10$ $\mathrm{mM} \mathrm{KCl}, 3 \mathrm{mM} \mathrm{CaCl}$ ). Two cell lines, AX2 (referred to as wild-type in this paper and an axenic derivative of the wild-type strain NC4) expressing GFPABD120k (24) and myosin heavy chain null cells (HS1) expressing GFP-myosin II (25), were used. ABD120k is the actinbinding domain of an actin cross-linking protein, ABP120k. ABD120k can bind to filamentous but not globular actin. Therefore, fluorescence imaging of GFPABD120k reflects the distribution of filamentous actin. The GFP-ABD120k gene was kindly provided from David A. Knecht (University of Connecticut, Storrs, $\mathrm{CT})$. This fused gene was inserted into the pBIG expression vector by Taro Q. Uyeda (AIST, Tsukuba, Japan). 


\section{Preparation of Elastic Substrata}

To simultaneously observe the dynamics of molecular machinery and traction forces in a migrating cell, we combined TIRF and force microscopy. To create an evanescent field above the surface of elastic substrata, the refractive index of the substrata must be higher than 1.33 , the index of the medium surrounding the cells. An evanescent field could not be created on the surface of elastic substrata made with previously described materials such as polyacrylamide (15) and gelatin (26) because of their low refractive indexes and/or low degree of transparency. Therefore, we selected a pair of liquid silicones (CY52-276A and B; Dow Corning Toray, Tokyo, Japan) as materials for the elastic substrata. These are platinum-catalyzed silicones that use a platinum complex to participate in hydrosilylation of a vinyl functional siloxane polymer by a hydride functional siloxane polymer. The gel made from the pair of silicones is clear and colorless, and its refractive index is 1.40 . Moreover, the elasticity of the gel can be modulated by changing the mixing ratio of the two liquid silicones.

A 300-mg aliquot of CY52-276A and a 250-mg aliquot of CY52-276B were mixed. The mixture was spread on a 22 $\times 22$ mm coverslip (No. 1; Matsunami, Osaka, Japan) using a glass rod. The thickness of the silicone layer was about $10-20 \mu \mathrm{m}$. The silicone mixture on the coverslip was allowed to solidify at room temperature $\left(23^{\circ} \mathrm{C}\right)$ for two days. The solidified substrata were kept in a hermetically sealed case with an $100-\mu \mathrm{L}$ aliquot of a liquid silane (3-aminopropyl triethoxysilan; Sigma-Aldrich Japan, Tokyo, Japan) at $70^{\circ} \mathrm{C}$ for $1 \mathrm{~h}$ in order to attach the silan to the surface of the silicone substrata by vapor deposition. A round chamber $(16 \mathrm{~mm}$ in diameter and $2 \mathrm{~mm}$ in depth) was assembled using the coverslip on which the silicone substratum was spread at the bottom of the chamber. A $400-\mu \mathrm{L}$ aliquot of a solution containing fluorescent red microspheres ( $20 \mathrm{~nm}$ in diameter, the peak excitation and emission wavelengths were 580 and $605 \mathrm{~nm}$, respectively, F-8786; Molecular Probes, Invitrogen Japan, Tokyo, Japan) diluted 40,000 times with distilled water was added to the solidified silicone. After about $1 \mathrm{~min}$, the bead solution was
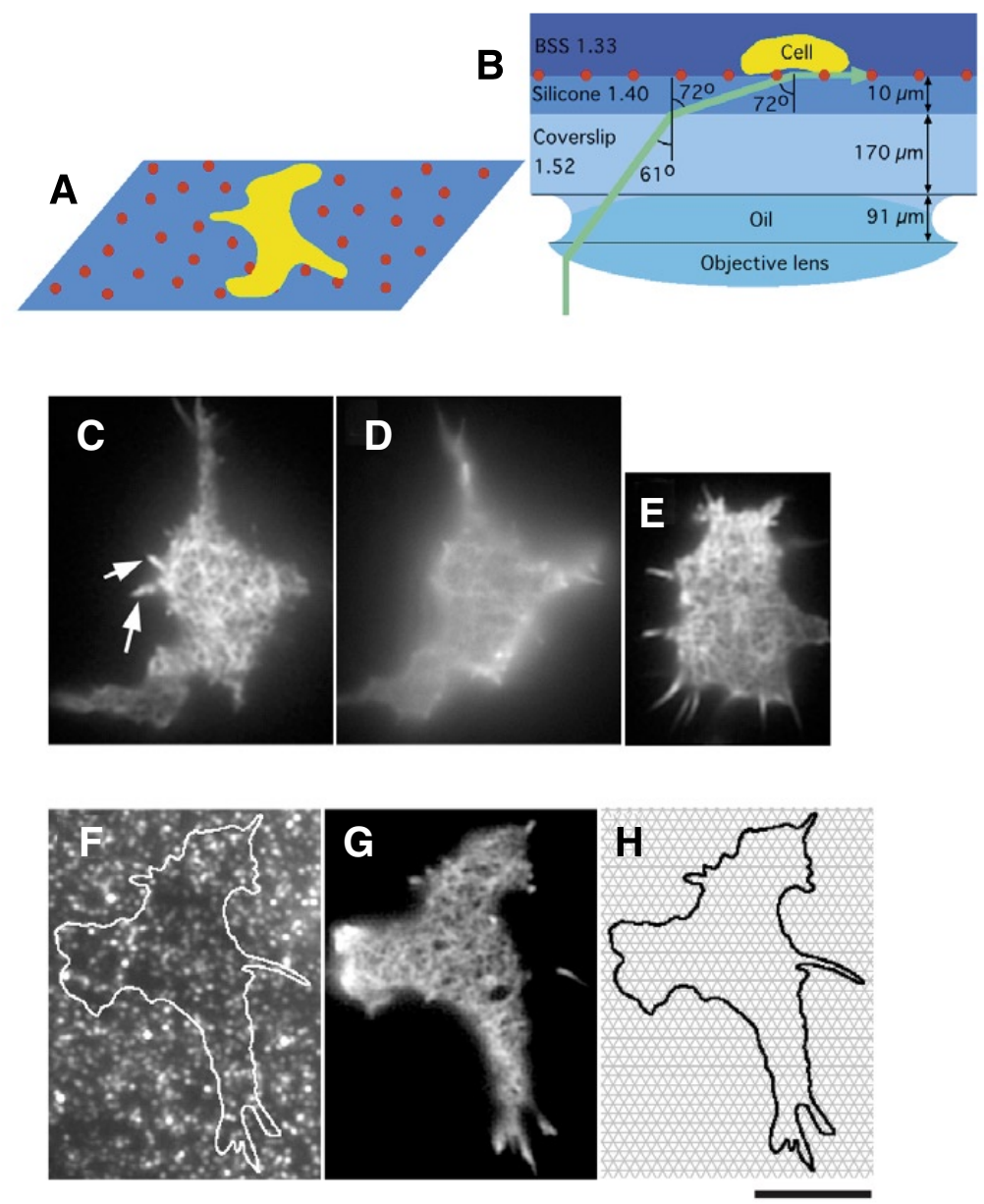

Figure 1. Combination of total internal refractive fluorescence (TIRF) and force microscopies. (A) Aggregation-competent Dictyostelium cells (yellow) were dispersed on an elastic substratum (blue) that was made from a pair of platinum-catalyzed liquid silicones with a high refractive index of 1.40 . Fluorescent red microspheres ( $20 \mathrm{~nm}$ in diameter; red filled circles) were embedded in the surface of the substratum beforehand. The thickness of the substratum was about $10 \mu \mathrm{m}$. The Young's modulus of the silicone substratum was typically $1.0 \mathrm{kPa}$. (B) A migrating cell (yellow) on silicone substratum embedded with fluorescent red microspheres (red small circles) was observed by TIRF microscopy with a $60 \times$ (NA 1.45) objective lens. The refractive indices of the coverslip, silicone substratum, and a balanced salt solution (BSS) were 1.52, 1.40, and 1.33, respectively. Refractive indices of the objective lens and immersion oil were the same as that of the coverslip. The thickness of the coverslip and silicone substratum were about $170 \mu \mathrm{m}$ and about $10 \mu \mathrm{m}$, respectively. A laser diode at a wavelength of $473 \mathrm{~nm}$ and an He-Ne laser at a wavelength of $543 \mathrm{~nm}$ were used for excitation of GFP and the red beads, respectively. The passed light of the lasers is indicated as a green line. To create an evanescent field above the surface of the silicone substrata, the angle of incidence to BSS from the silicone substratum should be $>72^{\circ}$. When the thickness of the substratum is $10 \mu \mathrm{m}$ and the angle of incidence to BSS is adjusted to $72^{\circ}$, the working distance of the objective lens should be about $91 \mu \mathrm{m}$. (C) A typical image of GFP-ABD120K in a Dictyostelium cell migrating on the silicone substratum. Actin filaments were clearly observed as is the case of the TIRF image of the cell attached to the glass coverslip (E), indicating that an evanescent field was created above the surface of the silicone substratum. Arrows in (C) indicate filopodia that are clearly observed as actin bundles. (D) Epifluorescence image of the same cell as (C) attached to the silicone substratum. (F) A typical image of fluorescent red beads concurrently detected with the image of GFP-ABD120K (G) in a Dictyostelium cell migrating on the silicone substratum. The white line in $(\mathrm{F})$ indicates the outline of the migrating cell. The positions of each bead are clearly distinguishable. $(\mathrm{H})$ Traction stress was calculated using the triangle finite element method. An image of the beads composed of $512 \times 512$ pixels was divided into approximately 8000 triangle elements. The length of the sides of each triangle was about $400 \mathrm{~nm}$ (equal to 8 pixels). Displacements of the nodes of each element were calculated from the displacements of the beads. The strains of each element were calculated from the displacements of its three nodes. The stresses of each element were calculated from the strains of the element under the assumption that the substrata are in a plane stress state. The magnitudes of the traction stresses under cells at each element were visualized using pseudocolor as shown in Figure 3, B, C, F, and Supplementary Movies S1 and S2. Scale bar, 4 $\mu \mathrm{m}$. GFP, green fluorescent protein. 


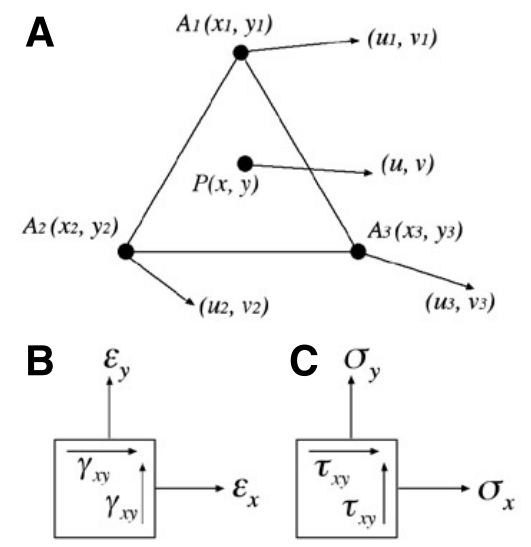

Figure 2. Traction stress was calculated in each triangle element. (A) Each triangle element has three nodes, $A_{1}, A_{2}$, and $A_{3}$, whose original positions are $\left(x_{1}, y_{1}\right),\left(x_{2}, 2_{2}\right)$, and $\left(x_{3}, y_{3}\right)$, and displacement vectors are $\left(u_{1}, v_{1}\right),\left(u_{2}, v_{2}\right)$, and $\left(u_{3}, v_{3}\right)$, respectively. The strains at each point in one element are assumed to be distributed linearly. $\mathrm{P}$ is an arbitrary point whose original position is $(x, y)$ and the displacement vector is $(u, v)$. (B) The longitudinal and shearing strains $\varepsilon_{\chi}, \varepsilon_{y}$, and $\gamma_{x y}$ at the minute square in the element. (C) The normal and shearing stresses $\sigma_{\chi}, \sigma_{y}$, and $\tau_{x y}$ at the minute square in the element.

aspirated off, and $400 \mu \mathrm{L}$ BSS were added.

The Young's modulus of the elastic substrata was measured using the method of Lo et al. (27). Briefly, a steel ball $\left(0.5 \mathrm{~mm}\right.$ diameter, $\left.7.8 \mathrm{~kg} / \mathrm{m}^{3}\right)$ was placed on a substratum embedded with fluorescent beads. The indentation caused by the steel ball was measured by following with the microscope-focusing mechanism the vertical position of the fluorescent beads under the center of the ball. The Young's modulus was calculated as $Y=3\left(1-p^{2}\right) f^{2} / 4 d^{3 / 2} r^{1 / 2}$, where $f$ is the force exerted on the sheet, $d$ is the indentation, $r$ is the radius of the steel ball, and $p$ is the Poisson ratio (assumed to be 0.5; see Reference 12). The Young's modulus of the silicone substrata was typically $1.0 \mathrm{kPa}$.

\section{Microscopy}

Migrating Dictyostelium cells containing a GFP-fused gene plated on a silicone substratum with fluorescent red beads on the surface (Figure 1A) were observed by TIRF microscopy using an Olympus IX71 inverted microscope
(Olympus, Tokyo, Japan) with a $60 \times$ objective lens (PLAPON 60× OTIRFM, NA 1.45). A laser diode (DPBL-9010F; Photop Technologies, Fujian, China) at a wavelength of $473 \mathrm{~nm}$ and an $\mathrm{He}-\mathrm{Ne}$ laser (Research Electro Optics, Boulder, Colorado, USA) at a wavelength of 543 $\mathrm{nm}$ were used for exciting of GFP and red beads, respectively. Exciting light from each laser was applied in turn by electromagnetic shutters (No. 0; Copal, Tokyo, Japan) in front of the laser heads.

Fluorescence of GFP and the red beads was detected with a dualband pass filter (XF3056; Chroma Technology, Rockingham, VT, USA). Fluorescence images were collected using a cooled charge-coupled device (CCD) camera (ORCA ER; Hamamatsu Photonics, Tokyo, Japan). The electromagnetic shutters and recording of the CCD camera were controlled with a custom program developed under LabVIEW 7.1 (National Instruments, Tokyo, Japan). Figure 1B shows an illustration of the vertical cross-section of the optical path. The light from each laser first passes through an area whose refractive index is 1.52 , including the objective lens, immersion oil, and a coverslip. Then it is refracted at the boundary between the coverslip and the silicone substratum, whose refractive index is 1.40. Finally, the light is reflected at the boundary between the silicone substratum and BSS standard saline for Dictyostelium cells $(10 \mathrm{mM}$ $\mathrm{NaCl}, 10 \mathrm{mM} \mathrm{KCl}, 3 \mathrm{mM} \mathrm{CaCl}$ ), whose refractive index is 1.33. To achieve total internal reflection at the boundary between the silicone substratum and BSS, the angle of incidence $(\varnothing)$ should be $>72^{\circ}(\sin \varnothing \geq 1.33 / 1.40)$.

To ascertain whether an evanescent field of excitation light was created above the silicone substratum, we observed the distribution of F-actin in a migrating Dictyostelium cell. F-actin was visualized by GFP-ABD $120 \mathrm{k}$ (24). A representative image of GFPABD120k is shown in Figure 1C. Actin meshwork was observed clearly, indicating that an evanescent field was created above the silicone substratum (28), as is the case of the TIRF image of the cell attached to the glass coverslip (see Figure 1E). Figure 1D shows the epifluorescence image of the same cell as Figure $1 \mathrm{C}$ attached to the silicone substratum. Fluorescent red beads at the top of the silicone layer that were detected concurrently with the GFPABD120k signal (Figure 1G) are shown in Figure 1F. The beads could be distinguished from each other.

\section{Calculation of Traction Stresses}

Traction forces were not calculated as the real values of the force in cells with the Newton scale, but as the stress in the substrate with the Pascal scale using the triangle finite element method (29). Migrating cells cause strains in the elastic substratum and displacements of the beads in the substratum. For each observation, a series of images of the beads and another reference image taken after the cell had been removed by pipeting were obtained. Displacements of the beads were calculated by comparing the positions of beads between each image with displaced beads and the reference image. A representative sample image of beads detected simultaneously with GFP-ABD120k (Figure 1G) is shown in Figure 1F.

An image of the beads composed of $512 \times 512$ pixels was divided into approximately 8000 triangle elements as shown in Figure 1H. The length of the sides of each triangle is about 400 $\mathrm{nm}$. Displacements of the nodes of each element were calculated as mean values of that of neighboring beads. The strains of each element were calculated from the displacements of its three nodes. The stresses of each element were calculated from the strains of the element under the assumption that the substratum is in a plane stress state. To use the plane stress approximation, the displacements of the beads in all experiments were kept $<1 \mu \mathrm{m}$, one-tenth of the thickness of the gel.

As shown in Figure 2A, each triangle element has three nodes $A_{l}$, $A_{2}$, and $A_{3}$, whose original positions are $A_{1}\left(x_{1}, y_{1}\right), A_{2}\left(x_{2}, y_{2}\right)$, and $A_{3}\left(x_{3}\right.$, $\left.y_{3}\right)$, and displacement vectors are $\left(u_{l}\right.$, $\left.v_{1}\right),\left(u_{2}, v_{2}\right)$, and $\left(u_{3}, v_{3}\right)$, respectively. The displacement vector $(u, v)$ of the arbitrary point $P$, whose original position is $(x, y)$ in the triangle element, is determined as: 


\author{
$\left(\begin{array}{l}u \\ v\end{array}\right)=N \cdot U_{m}$ \\ $N=\left(\begin{array}{cccccc}N_{1} & 0 & N_{2} & 0 & N_{3} & 0 \\ 0 & N_{1} & 0 & N_{2} & 0 & N_{3}\end{array}\right)$ \\ $U_{m}=\left(\begin{array}{llllll}u_{1} & v_{1} & u_{2} & v_{2} & u_{3} & v_{3}\end{array}\right)^{T}$ \\ $N_{1}=(\operatorname{det} A)^{-1}\left\{\left(x_{2} y_{3}-x_{3} y_{2}\right)+\left(y_{2}-y_{3}\right) x+\left(x_{3}-x_{2}\right) y\right\}$ \\ $N_{2}=(\operatorname{det} A)^{-1}\left\{\left(x_{3} y_{1}-x_{1} y_{3}\right)+\left(y_{3}-y_{1}\right) x+\left(x_{1}-x_{3}\right) y\right\}$ \\ $N_{3}=(\operatorname{det} A)^{-1}\left\{\left(x_{1} y_{2}-x_{2} y_{1}\right)+\left(y_{1}-y_{2}\right) x+\left(x_{2}-x_{1}\right) y\right\}$ \\ $\operatorname{det} A=x_{2} y_{3}+x_{1} y_{2}+x_{3} y_{1}-x_{1} y_{3}-x_{2} y_{1}-x_{3} y_{2}$
}

[Eq. 1]

In the above calculation, the strains at each point in the element are assumed to be distributed linearly. The longitudinal and shearing strains $\varepsilon_{x}, \varepsilon_{y}$, and $\gamma_{x y}$ at the minute square (Figure $2 \mathrm{~B}$ ) in each element are determined as:

$$
\varepsilon_{x}=\frac{\partial u}{\partial x}, \quad \varepsilon_{y}=\frac{\partial v}{\partial y}, \quad \gamma_{x y}=\frac{\partial u}{\partial y}+\frac{\partial v}{\partial x}
$$

The strain matrix:

$$
\left[\varepsilon_{m}\right]=\left(\begin{array}{lll}
\varepsilon_{x} & \varepsilon_{y} & \gamma_{x y}
\end{array}\right)^{T}
$$

is calculated with Equations 1 and 2 as follows:

$B=(\operatorname{det} A)^{-1}\left(\begin{array}{cccccc}y_{2}-y_{3} & 0 & y_{3}-y_{1} & 0 & y_{1}-y_{2} & 0 \\ 0 & x_{3}-x_{2} & 0 & x_{1}-x_{3} & 0 & x_{2}-x_{1} \\ x_{3}-x_{2} & y_{2}-y_{3} & x_{1}-x_{3} & y_{3}-y_{1} & x_{2}-x_{1} & y_{1}-y_{2}\end{array}\right)$

[Eq. 4]

The normal and shearing stresses $\sigma_{x}$, $\sigma_{y}$, and $\tau_{x y}$ at the minute square (Figure 2C) in each element are calculated from the strain matrix (Equation 3) under the assumption that the substratum is in a plane stress state.

$$
\begin{aligned}
& {\left[\sigma_{m}\right]=D\left[\varepsilon_{m}\right]} \\
& {\left[\sigma_{m}\right]=\left(\begin{array}{lll}
\sigma_{x} & \sigma_{y} & \tau_{x y}
\end{array}\right)^{T}} \\
& D=\frac{E}{1-v^{2}}\left(\begin{array}{ccc}
1 & v & 0 \\
v & 1 & 0 \\
0 & 0 & \frac{1-v}{2}
\end{array}\right)
\end{aligned}
$$

[Eq. 5]
In the above equations, $E$ and $v$ mean Young's modulus and Poisson ratio, respectively. In this study, the magnitude of stress $\left|\sigma_{m}\right|$ in each element is defined as:

$$
\left|\sigma_{m}\right|=\left\{\left(\sigma_{x}+\tau_{x y}\right)^{2}+\left(\sigma_{y}+\tau_{x y}\right)^{2}\right\}^{1 / 2}
$$

The magnitude of the stress is constant in one triangle element in our calculation because of the assumption of linear distribution of strains in one element as mentioned above.

Cell boundaries were drawn manually using light images or fluorescent imaging of GFP. The magnitudes of the traction stresses for each element under a cell were visualized with pseudocolor. The elements outside of the cell were filled with black, although the stresses there were not zero. Each node and the vector of its displacement are superimposed on the pseudocolor images as white circles and bars, respectively. Typical images of simultaneous visualization of traction force and the fluorescence of GFPABD120k, reflecting actin filaments, are shown in Figure 3, A and B, and in the Supplementary Movie S1 available online at www.BioTechniques.com. All of these calculations and a portion of the image processing were performed using a custom program developed under RealBASIC Ver. 3.5 (ASCII Solutions, Tokyo, Japan), and a portion of the image processing was performed using ImageJ Ver. 1.33 (freeware from $\mathrm{NIH}$ ), Image SXM Ver. 1.74 (a version of NIH Image, freeware from NIH), and Photoshop CS (Adobe, Tokyo, Japan).

\section{RESULTS AND DISCUSSION}

Using our new method, first we visualized the traction forces and the fluorescence of GFP-ABD120k, reflecting actin filaments, simultaneously (Figure 3, A and B, and Supplementary Movie S1). Large traction forces were detected at the region where fluorescence of GFP-ABD120k was high or at the tip of filopodia (Figure 3, A and B, arrowheads). The filopod is a finger-like extension of the cell surface composed of bundled actin filaments, and it presents an excellent model for actin-driven membrane protrusion. Dictyostelium cell produces filopodia not only to the substratum but also to the upper region of the cell. Under the TIRF illumination, only the filopodia extending to the substratum can be clearly observed. The traction forces generated by an actin bundle in a single filopod has never, to our knowledge, been measured using elastic substratum. Under our new microscopic technique, not only the actin meshwork but also actin bundles in filopodia were clearly observed in the evanescent field above the silicone layer (Figure 1C, arrows). Therefore, we first attempted to detect whether the actin bundles in filopodia exert counterforces of actin polymerization onto the substratum during extension and whether they exert contractile forces during contraction. The motility of an actin bundle in a filopod (Figure 3A, arrow) and its generating traction force were measured (Figure 3, C-E). The movement of the actin bundle in the filopod could be traced precisely as the fluorescence of GFP-ABD120k (Figure 3C, top). Interestingly, the filopod did not exert any force onto the substratum during its elongation period. On the other hand, the filopod exerted a traction force at the tip of the actin bundle during its contraction (Figure 3C, white arrow, and Supplementary Movie S2). The direction of the force was the same as that of the retraction. The length of the actin bundle and the stress in the substratum under the tip of the actin bundle over time are shown in Figure 3D. At the beginning of retraction, the stress began to increase. Then, at the middle of retraction, it reached a peak value. Immediately afterward, not only the stress but also the length of the actin bundle decreased quickly, suggesting that detachment of the filopod from the substratum took place. Figure 3E shows the distribution of fluorescence of GFP-ABD120k and the stress along the length of the actin bundle from the base to the tip (Figure 3E, yellow lines in top images) at $26 \mathrm{~s}$ in Figure 3C. The magnitude of stress reaches a peak at the tip of actin bundle (Figure 3E, gray area). This strongly suggests that the filopodia contact the substratum just after the completing of the elongation and begin to pull it to the cell.

Simultaneous recording of the GFPABD120k signal and the traction forces 
allowed us to generate a detailed map of the traction forces exerted by a single filopod. The spatiotemporal resolution of the method presented here, in which $400 \mathrm{~nm}$ equals the length of the sides of each triangle element, was sufficient for analysis.

Next, we tried to detect the traction forces related to the other molecules using the new microscopic technique. In migrating Dictyostelium cells and fibroblasts (7-11), the detachment of the rear of the cell from the substratum and its retraction is assumed to be induced by contraction via a myosin II-dependent process, but there has been no direct evidence. The method presented here allowed us to simultane-
A

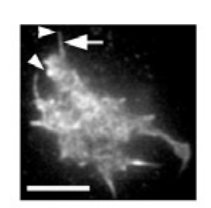

B
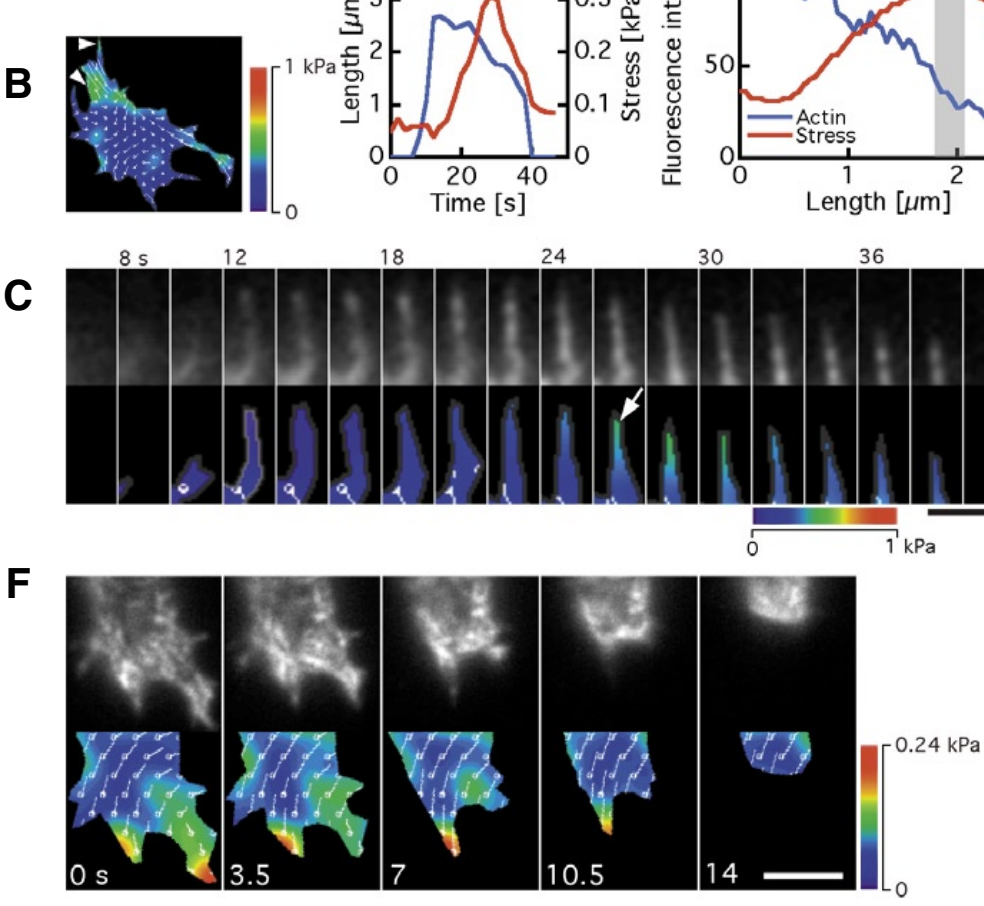

Figure 3. Simultaneous detection of traction forces and actin bundles or filamentous myosin II. (A) Fluorescent image of GFP-ABD120k in a migrating wild-type Dictyostelium cell transfected with GFPABD120k. Bar, $5 \mu \mathrm{m}$. (B) Traction force measurement concurrently visualized with the image of GFPABD120K (A). Large traction forces were detected at the region where fluorescence of GFP-ABD120k was high or at the tip of filopodia [arrowheads in (A) and (B)]. See Supplementary Movie S1 available online at www.BioTechniques.com. (C) Traction force measurement exerted by a single filopod [an arrow in (A)]. A typical result from five cells is shown. (Top) The movement of the actin bundle in the filopod was traced precisely as the fluorescence of GFP-ABD120k. (Bottom) The stress in the substratum reflects the traction force exerted by the filopod. During the elongation period, the filopod did not exert any traction force. On the other hand, it exerted a traction force at the tip of the actin bundle during contraction (arrow). The direction of the force was the same as that of the retraction. See Supplementary Movie S2. The time course is indicated on each picture. Bar, $2 \mu \mathrm{m}$. (D) Length of actin bundle in the filopod (blue) in (B) and stress in the substratum (red) under the tip of the actin bundle over time. The local traction stress was detected only at the tip of the actin bundle in the retracting filopod. (E) Distribution of the fluorescence of GFP-ABD120k (blue) and the stress in the substratum along the length of the filopod (red) from the base to the tip (yellow lines in top images) at $26 \mathrm{~s}$ in $(\mathrm{C})$. The value of the stress reached a peak at the tip of the actin bundle (gray area). (F) Simultaneous recording of GFP-myosin II (top) and traction forces (bottom) of a migrating myosin II null Dictyostelium cell expressing GFP-myosin II. The posterior region of a migrating Dictyostelium cell is shown. The directions of the strains of the substratum at each position depicted with white circles are indicated by white bars. The length of the white bars is three times as long as the magnitude of the strain. Accumulation of filamentous myosin II and large stresses are shown at the posterior end of the cell before contraction. A typical result from three cells is shown. Time course is indicated in each picture. Bar, $5 \mu \mathrm{m}$. GFP, green fluorescent protein. ously observe the dynamics of myosin II filaments and traction forces (Figure 3F). Myosin II filaments were observed using GFP-myosin II. As expected, accumulation of filamentous myosin II and high traction forces before retraction could be observed at the posterior of the cells. The direction of the stresses was the same as the direction of cell migration. This result strongly supports the assumption that the posterior retraction of migrating cells is induced by contraction via a myosin II-dependent process.

To understand the mechanism of cell migration, it is important to determine the spatiotemporal patterns of cellsubstratum mechanical interactions and their relationship to molecular dynamics. Several investigators have attempted to simultaneously observe traction forces and molecular dynamics (17,30-33). Using micromachined devices containing an array of closely spaced vertical microneedles, the relationships between molecular dynamics and traction forces were investigated (3033). Although the spatial resolution of these microdevices (several $\mu \mathrm{m}$ ) was not enough to depict a detailed force map, the obtained findings suggest that force distribution is spatially correlated to the localization of the focal adhesion protein vinculin (31). Correlation of the distribution of GFP-zyxin and traction forces has also been suggested based on measurements of the elastic substratum under normal fluorescence microscopy (17). Methods using elastic substrata should provide adequate spatial resolution for traction force measurements, although molecular distributions cannot be observed in detail under normal fluorescence microscopy. Here, we have described a combination of TIRF and force microscopies using an elastic substratum that provides enough spatial resolution (400 nm equals the length of the sides of each triangle element) to measure not only traction force but also molecular distribution (Figures 1 and 3). Further detailed observations using the new microscopy technique could shed light on the role of F-actin in elongation and retraction of filopodia and the role of myosin II in the retraction of the posterior ends of migrating cells. The new method described here could elucidate the relationships between the dynamics of molecules such as actin, myosin II, other subtypes of myosin, integrins, and cellular traction forces. 


\section{ACKNOWLEDGMENTS}

We thank Taro Q. Uyeda (AIST, Tsukuba, Japan) for the kind gift of the GFPABD120k expression plasmid and David A. Knecht (University of Connecticut, Storrs, CT, USA) for the permission to use it. This study was partly supported by a Grant-inAid for Scientific Research on Priority Areas (no.17049020) to S.Y. from the National Institutes of Health and a grant to Y.I. from the Yamaguchi University Foundation. We are grateful for their support.

\section{COMPETING INTERESTS STATEMENT}

The authors declare no competing interests.

\section{REFERENCES}

1. Ridley, A.J., M.A. Schwartz, K. Burridge, R.A. Firtel, M.H. Ginsberg, G. Borisy, J.T. Parsons, and A.R. Horwitz. 2003. Cell migration: integrating signals from front to back. Science 302:1704-1709.

2. Raftopoulou, M. and A. Hall. 2004. Cell migration: Rho GTPases lead the way. Dev. Biol. 265:23-32.

3. Parent, C.A. 2004. Making all the right moves: chemotaxis in neutrophils and Dictyostelium. Curr. Opin. Cell Biol. 16:4-13.

4. Wang, Y.L. 1985. Exchange of actin subunits at the leading edge of living fibroblasts: possible role of treadmilling. J. Cell Biol. 101:597-602.

5. Dent, E.W. and F.B. Gertler. 2003. Cytoskeletal dynamics and transport in growth cone motility and axon guidance. Neuron 40:209-227.

6. Pollard, T.D. and G.G. Borisy. 2003. Cellular motility driven by assembly and disassembly of actin filaments. Cell 112:453-465.

7. Yumura, S., H. Mori, and Y. Fukui. 1984. Localization of actin and myosin for the study of ameboid movement in Dictyostelium using improved immunofluorescence. J. Cell Biol. 99:894899.

8. Small, J.V. 1989. Microfilament-based motility in non-muscle cells. Curr. Opin. Cell Biol. 1:75-79.

9. Galbraith, C.G. and M.P. Sheetz. 1997. A micromachined device provides a new bend on fibroblast traction forces. Proc. Natl. Acad. Sci. USA 94:9114-9118.

10. Jay, P.Y. and E.L. Elson. 1992. Surface particle transport mechanism independent of myosin II in Dictyostelium. Nature 356:438-440.
11. Uchida, K.S., T. Kitanishi-Yumura, and $\mathbf{S}$ Yumura. 2003. Myosin II contributes to the posterior contraction and the anterior extension during the retraction phase in migrating Dictyostelium cells. J. Cell Sci. 116:51-60.

12. Dembo, M., T. Oliver, A. Ishihara, and K. Jacobson. 1996. Imaging the traction stresses exerted by locomoting cells with the elastic substratum method. Biophys. J. 70:2008-2022.

13. Dembo, M. and Y.L. Wang. 1999. Stresses at the cell-to-substrate interface during locomotion of fibroblasts. Biophys. J. 76:2307-2316.

14. Beningo, K.A., M. Dembo, I. Kaverina, J.V. Small, and Y.L. Wang. 2001. Nascent focal adhesions are responsible for the generation of strong propulsive forces in migrating fibroblasts. J. Cell Biol. 153:881-888.

15. Munevar, S., Y. Wang, and M. Dembo. 2001. Traction force microscopy of migrating normal and $\mathrm{H}$-ras transformed $3 \mathrm{~T} 3$ fibroblasts. Biophys. J. 80:1744-1757.

16. Munevar, S., Y.L. Wang, and M. Dembo. 2001 Distinct roles of frontal and rear cell-substrate adhesions in fibroblast migration. Mol. Biol. Cell 12:3947-3954.

17. Beningo, K.A., M. Dembo, I. Kaverina, J.V. Small, and Y.L. Wang. 2001. Nascent focal adhesions are responsible for the generation of strong propulsive forces in migrating fibroblasts. J. Cell Biol. 153:881-888.

18. Munevar, S., Y.L. Wang, and M. Dembo. 2004. Regulation of mechanical interactions between fi-

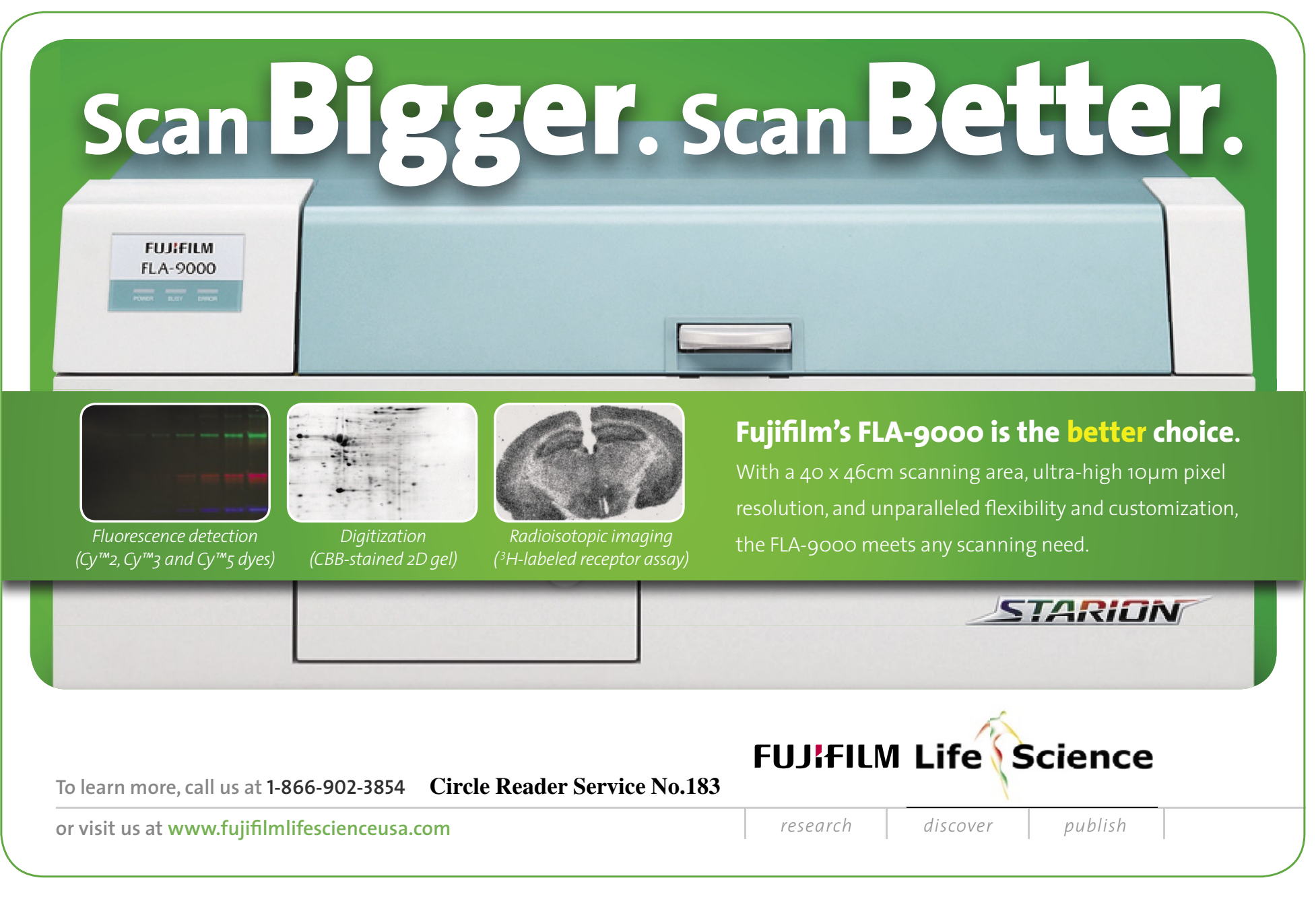


broblasts and the substratum by stretch-activated $\mathrm{Ca}^{2+}$ entry. J. Cell Sci. 117:85-92.

19. Shiu, Y.T., S. Li, W.A. Marganski, S. Usami, M.A. Schwartz, Y.L. Wang, M. Dembo, and S. Chien. 2004. Rho mediates the shear-enhancement of endothelial cell migration and traction force generation. Biophys. J. 86:2558-2565.

20. Butler, J.P., I.M. Tolic-Nørrelykke, B. Fabry, and J.J. Fredberg. 2001. Traction fields, moments, and strain energy that cells exert on their surroundings. Am. J. Physiol. Cell Physiol. 282: 595-605.

21. del Alamo, J.C., R. Meili, B. Alonso-Latorre, J. Rodriguez-Rodriguez, A. Aliseda, R.A. Firtel, and J.C. Lasheras. 2007. Spatio-temporal analysis of eukaryotic cell motility by improved force cytometry. Proc. Natl. Acad. Sci. USA 104:13343-13348.

22. Tsujioka, M., K. Yoshida, and K. Inouye. 2004. Talin B is required for force transmission in morphogenesis of Dictyostelium. EMBO J. 23:2216-2225

23. Lombardi, M.L., D.A. Knecht, M. Dembo, and J. Lee. 2007. Traction force microscopy in Dictyostelium reveals distinct roles for myosin II motor and actin-crosslinking activity in polarized cell movement. J. Cell Sci. 120:1624-1634.

24. Pang, K.M., E. Lee, and D.A. Knecht. 1998. Use of a fusion protein between GFP and an actin-binding domain to visualize transient filamentous-actin structures. Curr. Biol. 8:405-408.

25. Yumura, S. and T.Q. Uyeda. 1997. Transport of myosin II to the equatorial region without its own motor activity in mitotic Dictyostelium cells. Mol. Biol. Cell 8:2089-2099.

26. Doyle, A.D. and J. Lee. 2002. Simultaneous, real-time imaging of intracellular calcium and cellular traction force production. BioTechniques 33:358-364.

27. Lo, C.M., D.B. Buxton, G.C. Chua, M. Dembo, R.S. Adelstein, and Y.L. Wang. 2004. Nonmuscle myosin IIb is involved in the guidance of fibroblast migration. Mol. Biol. Cell 15:982-989.

28. Bretschneider, T., S. Diez, K. Anderson, J. Heuser, M. Clarke, A. Müller-Taubenberger, J. Köhler, and G. Gerisch. 2004. Dynamic actin patterns and Arp $2 / 3$ assembly at the substrate-attached surface of motile cells. Curr. Biol. 14:110.

29. Yoshino, M. 2002. The Guide of Finite Element Method with Excel, p. 38-54. In K. Asakura (Ed.), Asakura, Tokyo, Japan.

30. Balaban, N.Q., U.S. Schwarz, D. Riveline, P. Goichberg, G. Tzur, I. Sabanay, D. Mahalu, S. Safran, et al. 2001. Force and focal adhesion assembly: a close relationship studied using elastic micropatterned substrates. Nat. Cell Biol. 3:466472.

31. Tan, J.L., J. Tien, D.M. Pirone, D.S. Gray, K. Bhadriraju, and C.S. Chen. 2003. Cells lying on a bed of microneedles: an approach to isolate mechanical force. Proc. Natl. Acad. Sci. USA 100:1484-1489.

32. du Roure, O., A. Saez, A. Buguin, R. Austin, P. Chavrier, P. Silberzan, and B. Ladoux. 2005.
Force mapping in epithelial cell migration. Proc Natl. Acad. Sci. USA 102:2390-2395.

33. Sniadecki, N.J. and C.S. Chen. 2007. Microfabricated silicone elastomeric post arrays for measuring traction forces of adherent cells, p. 313-327. In Y.L. Wang and D. Discher (Eds.), Cell Mechanics. Methods in Cell Biology, vol. 83. Elsevier, Cambridge, MA.

Received 28 August 2007; accepted 19 December 2007.

Address correspondence to Yoshiaki Iwadate, Department of Functional Molecular Biology, Graduate School of Medicine, Yamaguch University, Yamaguchi 753-8512, Japan. e-mail:iwadate@yamaguchi-u.ac.jp

To purchase reprints of this article, contact: Reprints@BioTechniques.com
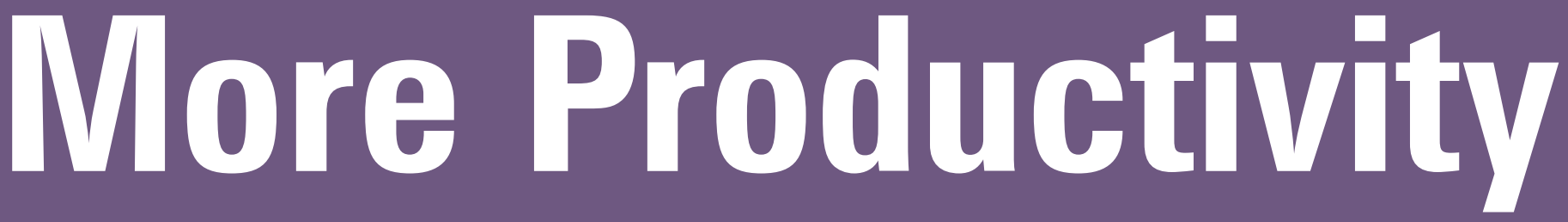

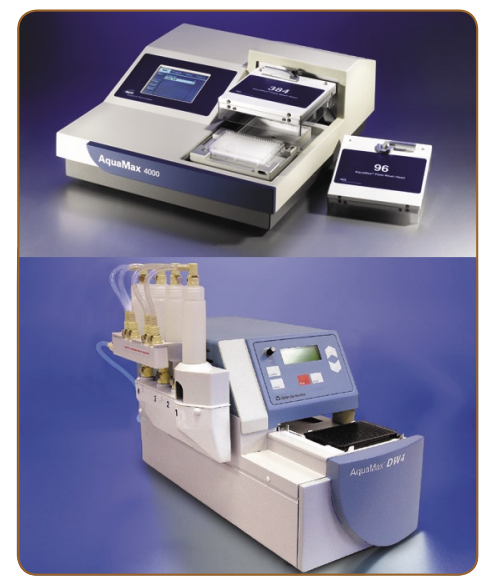

The flexible 96/384-well AquaMax 4000 washer (top) and integrated 96/384/1536-well AquaMax DW4 washer/ dispenser (bottom)
Run more assays in less time with the new AquaMax ${ }^{\circledR}$ series of washers and dispensers from MDS Analytical Technologies. With a unique modular design, the AquaMax systems can be easily configured to meet your current biochemical and cell-based application requirements, providing affordable upgrade options when your lab requirements change. The AquaMax 96-, 384-, and 1536-well heads are interchangeable and extend the capabilities of your washer and dispenser within a single instrument platform.

\section{More Flexibility}

$\Theta$ Configurable for 96-, 384-, and 1536-well microplate formats

$\Theta$ Up to 4 fluid inlets for buffers and solutions allow multiple users without bottle changing

$\Theta$ Integrated stacker option for up to 50 microplates

More Reliability

$\Theta$ Precise aspiration and dispense control allow fast microplate processing

$\oplus$ Comprehensive, single-button, OneTouch cleaning, prime, and rinse utilities

$\oplus$ Stores up to 99 user-defined programs

Upgrade your liquid handling tools to increase your productivity. Call us for an on-site demonstration or visit www.moleculardevices.com/liquid for more information. 